\title{
Growth Rate Patterns of the So-called Osmophilic and Non-osmophilic Yeasts in Solutions of Polyethylene Glycol
}

\author{
By J. C. ANAND AND A. D. BROWN \\ Department of Microbiology, University of New South Wales, \\ Kensington, Sydney, Australia
}

(Accepted for publication 8 December 1967)

\begin{abstract}
SUMMARY
The growth rates of nine yeasts, conventionally classified as 'osmophilic' and six yeasts, conventionally classified as 'non-osmophilic' were measured at $30^{\circ}$ in media adjusted to known water activities with polyethylene glycol. The organisms were less tolerant of low water activities $\left(a_{w}\right)$ in the presence of polyethylene glycol than in the presence of sugars. There was no significant difference between average minimal water activities supporting growth of the two groups of yeasts in polyethylene glycol, although differences in sugar tolerance were conspicuous. There was good correlation, however, between quantitative water relations of the organisms in polyethylene glycol and qualitative observations of ability to grow at specified sugar concentrations. The osmophilic organisms grew about half as fast as the non-osmophilic organisms at their respective optimal water activities. The osmophils had relatively broad water activity optima; the non-osmophils had sharp optima. Neither group of yeasts had a general requirement for a water activity lower than that of the basal medium $\left(0.997 a_{w}\right)$ in order to grow, although there were two osmophilic strains which would not grow in the basal medium at $30^{\circ}$. The adjective 'osmophilic' is considered to be inaccurate; until the physiology of these yeasts is better understood we propose that they be designated simply as 'sugar tolerant'. The quantitative results have provided a basis for selecting sugar tolerant organisms for biochemical and physiologica] investigations.
\end{abstract}

\section{INTRODUCTION}

The ability of an organism to thrive in a relatively dry environment is potentially subject to determination by its response to high solute activity, low solvent activity and, less obviously, possible effects of osmotic pressure. The halophilic bacteria exemplify a group of organisms with a strict requirement for high solute activity, the dominant solutes in this case being the ions $\mathrm{Na}^{+}$and $\mathrm{Cl}^{-}$. The requirement for $\mathrm{Na}^{+}$is related to some distinctive features of halophilic bacterial proteins (Brown, 1964). There are at least two other groups of micro-organisms which can thrive in a dry environment which need not necessarily have a high ionic strength. These organisms are the so-called 'osmophilic' yeasts and 'xerophilic' moulds (Scott, I956).

The osmophilic yeasts are distinguished by their ability to grow in highly concentrated sugar solutions and it is a common practice to discuss the behaviour of such yeasts in terms of sugar tolerance; occasionally they will tolerate relatively concentrated solutions of $\mathrm{NaCl}$. The osmophilic yeasts are the subject of quite a substantial 
literature of which the reviews by Scott (1956), Ingram (1957) and Onishi (1963) are recent and useful. In spite of a substantial literature, however, there is currently no information about the physiological or biochemical basis of their tolerance of concentrated sugar solutions. The designation 'osmophilic', which was proposed by Richter (I9I2), is itself unfortunate because there is nothing at present to justify the implication that osmotic pressure is a factor in determining the peculiar physiology of these organisms. Scott (1956) has also commented on the disadvantages of interpreting the water relations of osmophilic yeasts in terms of osmotic pressure.

The current state of information is such that it is difficult, if not impossible, to describe the condition of 'osmophilia' in other than qualitative or loose quantitative terms. There is no systematic information relating growth rates to water activity as distinct from sugar concentration. This is not necessarily a disadvantage in seeking a definition, but it is no help in understanding the biological phenomenon involved. The literature leaves little doubt, however, that 'osmophilia' is a genuine phenomenon which warrants investigation as a means of understanding the ability of organisms to thrive in dry environments. The present paper describes the first stage of such an investigation and, in part, is an attempt to define osmophilic yeasts in terms of growth rates and water activities. Growth rates of fifteen yeasts were determined in media adjusted to known water activities with polyethylene glycol. This solute was selected in order to minimize the complication of large and varying concentrations of ionizing metabolites which accumulate when water activity is adjusted with sugars and also, it was found, with the monomer ethylene glycol. The classification of the yeasts as osmophilic or otherwise based on growth rate measurements agreed with the classification based on sugar tolerance. The growth rate patterns showed that, in general, osmophilia is a tolerance of, rather than a requirement for, dry conditions and allow some tentative generalizations to be made.

\section{METHODS}

Organisms. Nine yeasts, previously classified as osmophilic and six, previously classified as non-osmophilic, were obtained from sources shown in Table I. Yeasts, other than those which were obtained from the Centraalbureau voor Schimmelcultures, were identified by conventional diagnostic methods (Lodder \& Kreger-van Rij, 1952). The sugar fermentation and assimilation characteristics of Centraalbureau cultures were checked and confirmed in each case. The sugar tolerance of each yeast culture was ascertained by incubating the organisms in a series of sugar media at $30^{\circ}$ for up to 2 months (see Scarr \& Rose, 1966). Stock cultures of the osmophilic organisms were maintained on 'Synthetic Honey Agar' (yeast-extract agar plus $48 \%$ (w/v) glucose) at $4^{\circ}$. The non-osmophils were maintained on malt agar at $4^{\circ}$.

Growth medium. The organisms were grown, for experimental purposes, in a basal medium supplemented with polyethylene glycol as specified. The basal medium had the following composition: Peptone (Difco), 5.0 g.; yeast extract (Difco), 2.5 g.; $\mathrm{KH}_{2} \mathrm{PO}_{4}, \mathrm{I} \cdot 0 \mathrm{~g}$.; $\mathrm{MgSO}_{4} \cdot 7 \mathrm{H}_{2} \mathrm{O}, 0.25 \mathrm{~g}$.; $\mathrm{CaCl}_{2}, 0 . \mathrm{I} \mathrm{g}$.; glucose, I.9 g.; water, I 1. The water activity $\left(a_{w}\right)$ of this medium is approximately 0.997. Polyethylene glycol with an average molecular weight of 200 (Carbowax-200) was de-ionized on a column of mixed-bed ion exchange resin (Elgastat) and added to the basal medium to give the required water activity. The water activity was interpolated from a water sorption 


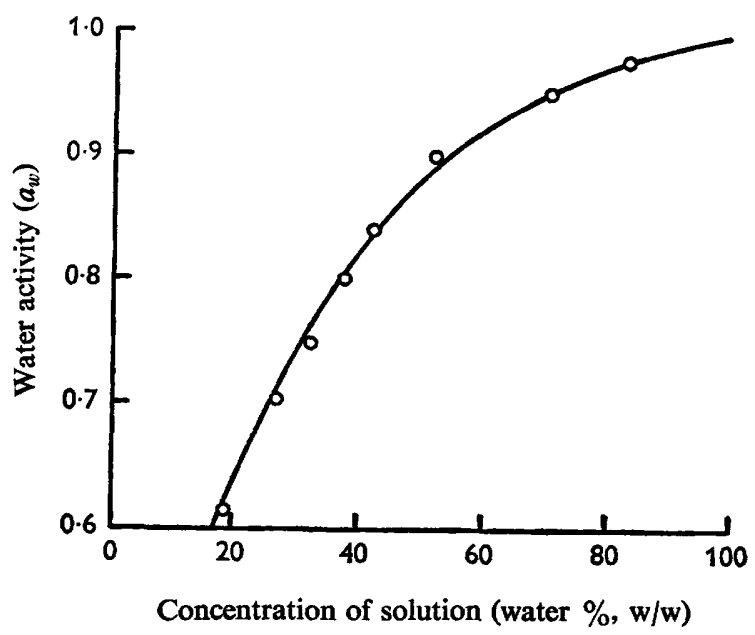

Fig. I. The composition of aqueous polyethylene glycol solutions of known water activity at $30^{\circ}$.

Table I. Description and origin of yeasts used Code
number

YA

YB

YC

YD

YE

YF

YG

YH

YK

Y 14

$\mathrm{Y}_{14}$

$\mathrm{Y}_{3} \mathrm{I}$

$\mathrm{Y} 34$

$Y_{40}$

$\mathrm{Y}_{4} \mathrm{I}$

$Y_{43}$
Name

Osmophilic strains

Saccharomyces rouxii

Zygosaccharomyces rugosus

(CBS 74I)

S. rouxii Boutroux var.

polymorphus (CBS IO9I)

$S$. mellis (CBs 736)

$Z$. nectarophilus (CBS 740)

$Z$. nussbaumeri (CBS 738)

$S$. rosei (CBS I090)

Torulopsis halonitratophila

(CBS 5240)

S. acidifaciens

Non-osmophilic strains

S. fragilis

S. cerevisiae

S. cerevisiae

S. cerevisiae

S. cerevisiae

S. cerevisiae
Source

Indian conserves

C.B.S.*

C.B.S.

C.B.S.

C.B.S.

C.B.S.

C.B.S.

C.B.S.

University of New South Wales

University of New

South Wales

University of New

South Wales

Brewery

Royal North Shore

Hospital

Baker's yeast

Brewer's Bottom Yeast

* Centraalbureau voor Schimmelcultures, Delft, Holland.

isotherm determined by equilibration against saturated salt solutions of known water activities between 0.62 and 0.98 (Robinson \& Stokes, 1949). This isotherm is shown in Fig. I.

Determination of growth rates. The organisms were grown in $40 \mathrm{ml}$. medium con- 
tained in Erlenmeyer flasks (capacity $250 \mathrm{ml}$.) fitted with cylindrical side arms, and plugged with cotton wool. Under conditions causing very low growth rates, flasks were also covered with polyethylene sheeting to minimize evaporation. The flasks were incubated in a horizontal platform shaker (70 oscillations/min.) at $30^{\circ}$. Growth rates were determined turbidimetrically at $700 \mathrm{~m} \mu$ with reference in each case to a sterile solution of the same composition as the growth medium. In preliminary determinations, turbidity was calibrated against total count of organisms and found, during exponential growth, to maintain a constant relation with count at all values of water activity down to 0.92 . The average standard deviation of growth rate measurements was 0.054 generations/hr (or, for mean generation time, $0.36 \mathrm{hr}$ ).

\section{Table 2. Growth of yeasts at specified sugar concentrations}

\begin{tabular}{|c|c|c|c|c|c|}
\hline \multirow[b]{3}{*}{ Yeast } & \multicolumn{4}{|c|}{ Concentration of sugar in medium $(\%, w / v)$} & \\
\hline & \multirow{2}{*}{$\begin{array}{c}\text { Fructose } \\
70\left(0.765 a_{w}\right)\end{array}$} & \multicolumn{4}{|c|}{ Sucrose } \\
\hline & & $65\left(0.865 a_{w}\right)$ & $60\left(0.895 a_{w}\right)$ & $55\left(0.917 a_{w 0}\right)$ & $50\left(0.935 a_{w}\right)$ \\
\hline \multicolumn{6}{|c|}{ Osmophilic } \\
\hline YA & + & + & + & & \\
\hline YB & + & + & + & & \\
\hline YC & + & + & + & & \\
\hline YD & + & + & + & & \\
\hline YE & + & + & + & & \\
\hline YF & + & + & + & & \\
\hline YG & - & + & + & & \\
\hline YH & + & + & $t^{*}$ & & \\
\hline YK & + & + & + & & \\
\hline \multicolumn{6}{|c|}{ Non-osmophilic } \\
\hline YI4 & & - & - & - & + \\
\hline $\mathbf{Y}_{3 \mathbf{I}}$ & & - & - & + & + \\
\hline$Y_{34}$ & & - & + & + & + \\
\hline$Y_{40}$ & & - & - & + & + \\
\hline $\mathbf{Y}_{41}$ & & - & - & - & + \\
\hline Y 43 & & - & - & + & + \\
\hline
\end{tabular}

Negative results were recorded after incubation had continued for at least 2 months at $30^{\circ}$. A blank space implies that the observation was not made.

* Yeast YH did not grow at $0.997 a_{w}$, nor at 0.990 in the presence of glycerol or sucrose; see Table 3 and compare Onishi (1960).

\section{RESULTS AND DISCUSSION}

Table 2 lists the ability of the organisms to grow in the basal medium plus sucrose or fructose at the specified concentration. The table enables the organisms to be classified into two groups, osmophilic and non-osmophilic, according to the criteria used by Scarr \& Rose (I966). Only one of the osmophils (YG) would not grow in sugar media at a water activity lower than 0.865 (equivalent to $65^{\circ}$ 'Brix', see Scarr $\&$ Rose, 1966). None of the osmophils grew in $75 \%$ fructose $\left(0 \cdot 700 a_{w}\right)$. None of the nonosmophils grew in media more concentrated than $60 \%(\mathrm{w} / \mathrm{w})$ sucrose $\left(0.895 a_{w}\right)$ and only one (Y 34$)$ grew above $55 \%(w / w)$ sucrose $\left(0.917 a_{w}\right)$.

Figures 2 and 3 show, for all the organisms, the relations between growth rate and water activity in media containing polyethylene glycol. Under these conditions the minimal water activities at which growth occurred were higher than in media adjusted 


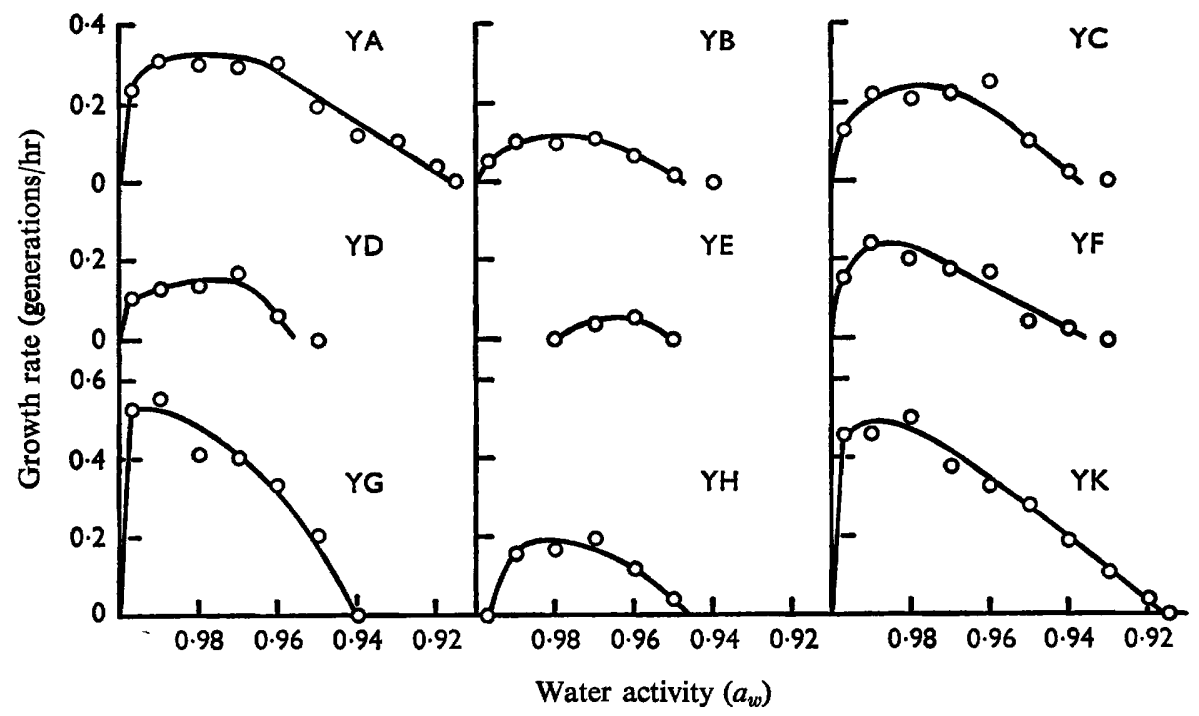

Fig. 2. Growth rate against water-activity relations; osmophilic yeasts in polyethylene glycol. The average standard deviation of the growth rate measurement was 0.054 generation/hr.

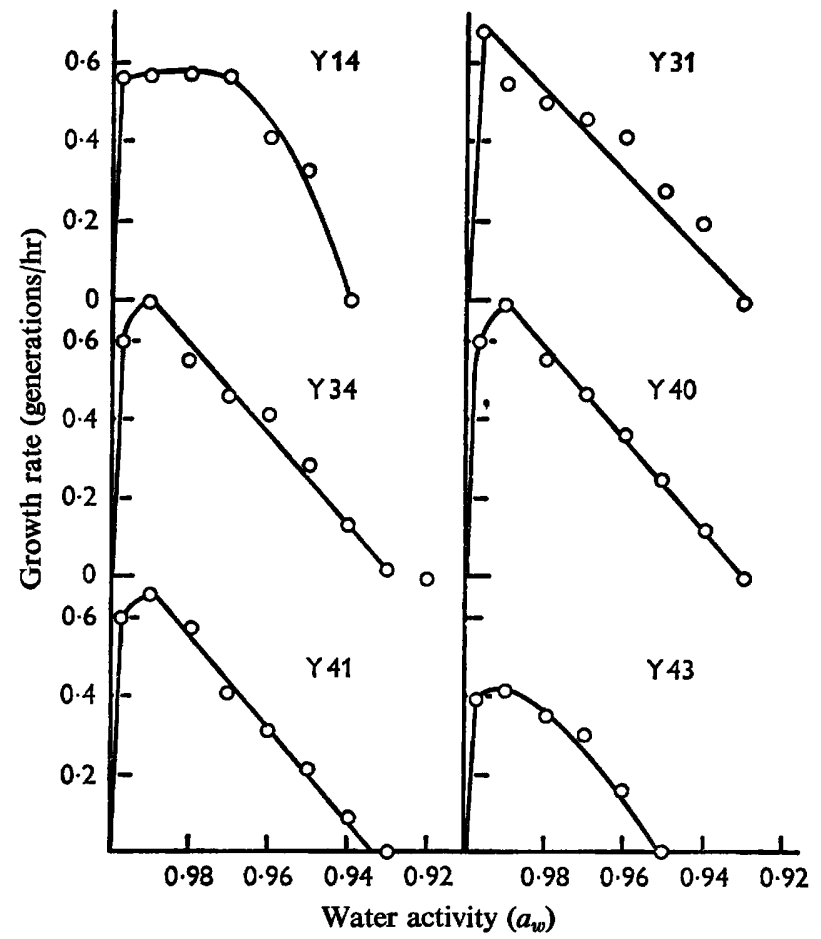

Fig. 3. Growth rate against water-activity relations; non-osmophilic yeasts in polyethylene glycol. The average standard deviation of the growth rate measurement was 0.054 generation/hr. 
with sugars. There was no significant difference between the average minimal water activity tolerated by the two groups of organisms, although the yeasts which grew at the lowest activity $(0.920)$ were osmophils (YA, YK). The maximal growth rates of the non-osmophils were more than twice as great as those of all but two of the osmophils. The two exceptional organisms were YG and YK. Yeast YG was less sugar-tolerant than the other members of its group and is presumed to be an intermediate type; yeast YK was distinguished by a low minimal water activity. The relatively low maximal growth rate of the osmophils is evidently an intrinsic property of the organisms since it was not caused specifically by polyethylene glycol. Table 3 lists mean generation times at $0.990 a_{w}$ in media adjusted with polyethylene glycol, glycerol or sucrose. There was close agreement with all three media.

Table 3. Mean generation time of yeasts in media adjusted to $0.990 a_{w}$ separately by three different solutes

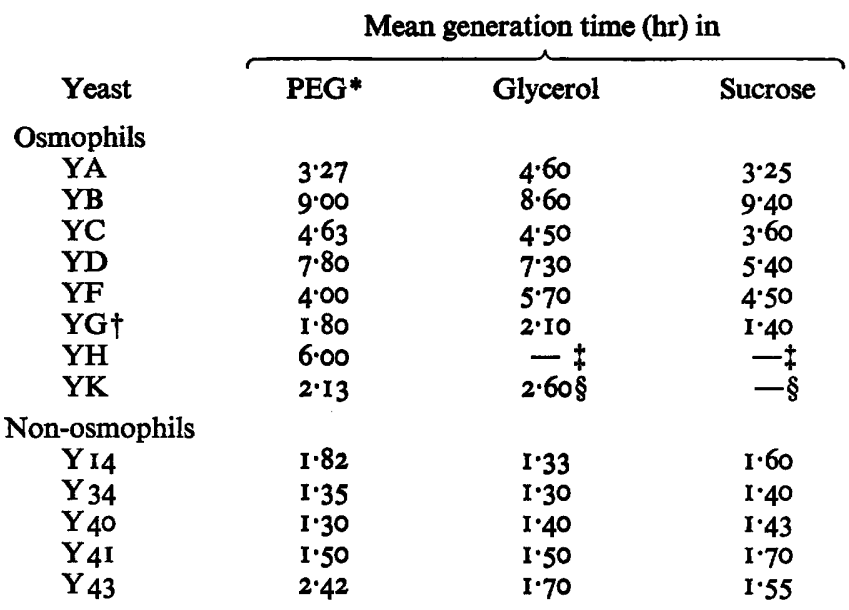

* Polyethylene glycol.

$\uparrow$ Probably an intermediate type; see text.

$¥$ No growth at this water activity in glycerol or sucrose.

$\S$ Organisms clumped making turbidimetric measurement difficult in glycerol and impossible in sucrose.

The optimal water activity of the non-osmophils (with one exception, Y I4) was confined to the narrow limits of 0.990-0.998; growth rates decreased sharply when water activity was decreased below the optimum. The exceptional yeast, Y 14, was distinguished also by poor tolerance of decreased water activity, both in polyethylene glycol and sugar solutions (Table I). The osmophils (except YG), on the other hand, had a broad optimum which extended in some cases down to $0.960 a_{w}$. Decreasing water activity below the optimal value caused a decline in growth rate which was appreciably less steep than that of the non-osmophilic yeasts. With two exceptions, the osmophils did not require a decreased water activity and grew well on the basal medium $\left(0.997 a_{w}\right)$. The exceptions were YE and YH. YE grew at measurable rates only within the range $0.970-0.960 a_{w}$. The shape of this curve does not necessarily imply that the organism specifically requires a decreased water activity; the curve shows what is evidently the optimal water activity in a poor medium. A more suitable 
medium (which was not sought) might be expected to raise the general values of growth rate to give a relation more closely resembling those of the other osmophilic organisms.

Yeast YH was a more interesting exception. It did not grow in the basal medium at $0.997 a_{w}$ nor in the presence of sucrose or glycerol at $0.990 a_{w}$; it did grow at the latter water activity, however, in the presence of polyethylene glycol. The organism, Torulopsis halonitratophila was described by Onishi (1960) as obligately halophilic (sic) at $30^{\circ}$, but facultatively so at $20^{\circ}$. The solute effects at $0.990 a_{w}$, as a first approximation, seem likely to be related to differences in solute penetration with associated effects on intracellular ionic strength. This suggestion can be tested experimentally; we hope to discuss it in due course.

There is thus no evidence that the osmophilic yeasts examined have a general requirement for decreased water activity, although there is limited evidence that some might grow optimally under drier conditions than do non-osmophilic yeasts. This finding supports Onishi's (1963) doubts about the existence of obligately osmophilic organisms. The designation '-philic' is inaccurate and would be better substituted by '-tolerant'.

Table 4. Mean generation time of yeast $Y A$ in media adjusted with polyethylene glycols of three molecular weights

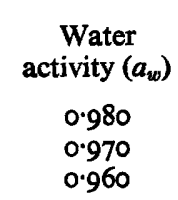

\begin{tabular}{ccc}
\hline PEG-200* & PEG-rooo* & PEG-4000* \\
3.50 & 3.70 & $4.40-5 \cdot 80$ \\
3.40 & 4.20 & $-t$ \\
3.40 & 3.80 & $-t$
\end{tabular}

* Polyethylene glycol of average molecular weight, 200, 1000 or 4000 .

$\uparrow$ Stated water activity not obtainable with PEG-4000.

The present results provide no information about the role of osmotic pressure in the physiology of these yeasts. Increased size and, therefore presumably, decreased penetration of polyethylene glycol molecules, however, decreased the growth rate of one yeast at high water activities (Table 4). Whether this effect was caused by differences is osmotic pressure or, as we believe, other physico-chemical consequences of solute penetration can be ascertained experimentally; it is the subject of another investigation. In the meantime we agree with Scott's (1956) comment that the osmophilic yeasts are better considered in terms of their water relations. We believe the designation 'osmophilic' to be misleading and we propose to refer to these organisms as 'sugar tolerant'.

Although simple measurements of growth rate give no information about physiological mechanisms they have allowed a phenomenon to be described quantitatively in terms which correlate satisfactorily with conventional qualitative statements of whether or not growth occurs at a specified sugar concentration. The quantitative description has also provided a rational basis for the selection of organisms for biochemical and physiological studies which are now in progress.

This work was supported by the Rural Credits Development Fund of the Reserve Bank of Australia. 


\section{REFERENCES}

Brown, A. D. (1964). Aspects of bacterial response to the ionic environment. Bact. Rev. $28,296$. InGRAM, M. (I957). Micro-organisms resisting high concentrations of sugars or salts. Symp. Soc. gen. Microbiol. 7, 90.

LodDer, J. \& KReger-VAN RIJ, N. J. W. (1952). The Yeasts. A Taxonomic Study. Amsterdam: NorthHolland Publishing Co.

ONISHI, H. (1960). Studies on osmophilic yeasts. IX. Isolation of a new obligate halophilic yeast and some consideration on halophilism. Bull. agric. chem. Soc. Japan 24, 226.

Onishi, H. (1963). Osmophilic yeasts. Advanc. Food Res. 12, 53.

RICHTER, A. A. von. (1912). Über einen osmophilen Organismus, den Hefepilz Zygosaccharomyces mellis acidi sp.n. Mykol. Zentbl. r, 67.

Robinson, R. A. \& Stokes, R. H. (I949). Water activities of saturated solutions at $25^{\circ}$ C. Ind. Engng Chem. 4r, 2013.

SCARR, M. P. \& Rose, D. (I966). Study of osmophilic yeasts producing invertase. J. gen. Microbiol. 45, 9.

Scotт, W. J. (I956). Water relations of food spoilage micro-organisms. Advanc. Food Res. 7, 83. 